\title{
Echocardiographic features of cardiac compression by a thymoma simulating cardiac tamponade and obstruction of the superior vena cava
}

\author{
MARIO I. CANEDO, LUTHER OTKEN, AND \\ MILTIADIS A. STEFADOUROS \\ From the Sections of Cardiology and Pathology, Medical College of Georgia, Augusta, \\ Georgia 30902, U.S.A.
}

$A$ patient with a large tumour of the superior mediastinum, detected by routine chest $\mathrm{x}$-ray examination, is presented. Compression of the heart and the superior mediastinum produced the clinical picture of cardiac tamponade and obstruction of the superior vena cava and trachea. The solid nature of the tumour was established by echocardiography which, in addition, showed: (1) posterior displacement of the heart; (2) diminished dimensions of the left atrium and left ventricle; (3) prominent respiratory variation in the position of the interventricular septum, the dimensions of both ventricles, and the duration of systolic separation of the aortic valve cusps; and (4) 'pseudo-prolapse' of the mitral valve. All abnormal clinical signs and echocardiographic findings disappeared promptly after surgical resection of the tumour which was found to be a thymoma.

Large 'tumours' of the anterior mediastinum occasionally produce clinical symptoms and physical signs suggestive of heart disease (Shaver et al., 1965; Allee et al., 1973; Peterson et al., 1975; Schloss et al., 1975). Though plain chest $x$-ray films can easily show their presence, more sophisticated radiological (Ferrané et al., 1970), angiocardiographic (Kincaid et al., 1960), or ultrasonic (Birnholz, 1973; Friday, 1973; Goldberg, 1973; Cardello et al., 1974; Peterson et al., 1975; Schloss et al., 1975) techniques are usually required to establish their extracardiac origin and to define their gross morphology further. B-scan ultrasonography has been successfully used to separate cystic from solid mediastinal masses (Friday, 1973; Peterson et al., 1975) but the very nature of this technique precludes assessment of the possible mechanical effects of the tumour on the function of the contracting heart. In two recent reports of the use of $\mathrm{M}$-mode echocardiography in the diagnosis of a large pericardial cyst (Peterson et al., 1975) or a cystic thymoma (Schloss et al., 1975), no echocardiographic evidence of mechanical interference of these 'tumours' with the function of the heart was noted. This report describes a patient with a large superior mediastinal tumour producing clinical manifestations of cardiac tamponade and obstruction of the superior vena cava, in whom both the gross nature of the tumour and its effect on the functional anatomy of the heart were studied by echocardiography.

\section{Case report}

The patient, a 17-year-old white youth, was admitted to the Eugene Talmadge Memorial Hospital on 20 May 1976. He had been in apparently good health until one month before admission when he began having progressive dyspnoea at rest, followed by dysphagia, dysphonia, and weight loss of $6.8 \mathrm{~kg}(15 \mathrm{lb})$. He also noticed distension of his neck and arm veins and swelling of the neck and face. On physical examination the patient was in moderate respiratory distress with inspiratory stridor and a respiratory rate of $28 / \mathrm{min}$. There was distinct venous distension with moderate oedema and cyanosis involving the head, neck, upper chest, and the arms. The jugular veins were distended up to the angle of the mandible in the sitting or standing position without any visible pulsations. The pulse rate was $88 / \mathrm{min}$ and showed sinus arrhythmia. The carotid and other peripheral arterial pulses were normal on expiration but very weak on inspiration; the blood pressure on expiration was $110 / 80 \mathrm{mmHg}$, with a $40 \mathrm{mmHg}$ pulsus paradoxus. The apex beat was not palpable. On auscultation the 


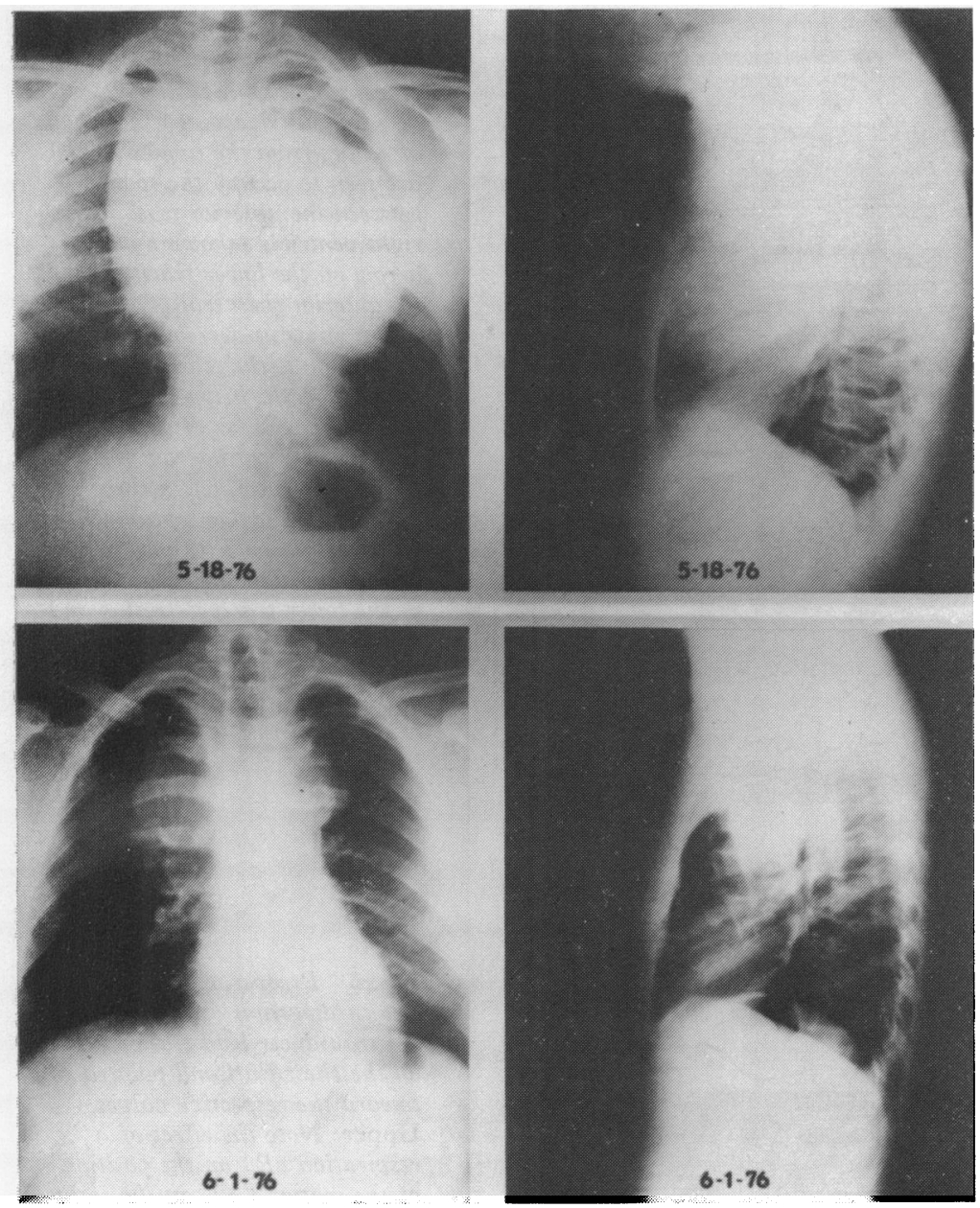

Fig. 1 Posteroanterior (left) and left lateral (right) chest film taken before (upper) and 11 days after surgical resection of the thymoma (lower). Note the remaining part of the tumour in the superior mediastinum in the right upper lung field. Also note posterior displacement of the heart by the tumour (upper right) with return of the heart in the normal anterior position after removal of the tumour (lower right).

heart sounds were normal; there were no murmurs or added sounds. Examination of the remainder of the systems was noncontributory. The haemoglobin was $15 \mathrm{~g} / \mathrm{dl}$ and the haematocrit 43 per cent. The electrocardiogram and other laboratory findings were normal. The chest $x$-ray film showed a large mass in the superior and anterior mediastinum; the heart was normal in size and configuration but displaced posteriorly (Fig. 1, upper).

\section{NONINVASIVE STUDY}

The M-mode echocardiogram, phonocardiogram, external carotid pulse, and respiratory tracing were simultaneously recorded on a strip chart, with the patient in a semi-reclining position. Fig. 2 shows a slow continuous $\mathrm{M}$-mode sector scan obtained from the fourth intercostal space parasternally. With the transducer directed superiorly and slightly medially, the aortic root could be seen at a depth of 5.5 to $8 \mathrm{~cm}$ from the posterior surface of the anterior chest wall, the intervening space being occupied by a homogeneous $4.5 \mathrm{~cm}$ wide band of echoes originating from the tumour $(T)$. These tumour echoes appeared as parallel lines of medium intensity with a small ( 1 to $2 \mathrm{~mm}$ ) amplitude motion whose directionanterior in systole and posterior in diastole-was similar to that of the aortic root, from which they were presumably transmitted. The anterior wall of the right ventricle was about $5 \mathrm{~cm}$ from the anterior chest wall in close proximity to the anterior aortic wall. The anteroposterior dimensions of the right ventricular outflow tract and the left atrium were diminished $(0.5$ to $0.1 \mathrm{~cm})$. The aortic valve echogram was normal but the duration of the ejection was abbreviated on inspiration. There was striking respiratory variation in the dimensions of the ventricles (Fig. 2, lower); thus, a prominent posterior displacement of the septum on inspiration was 

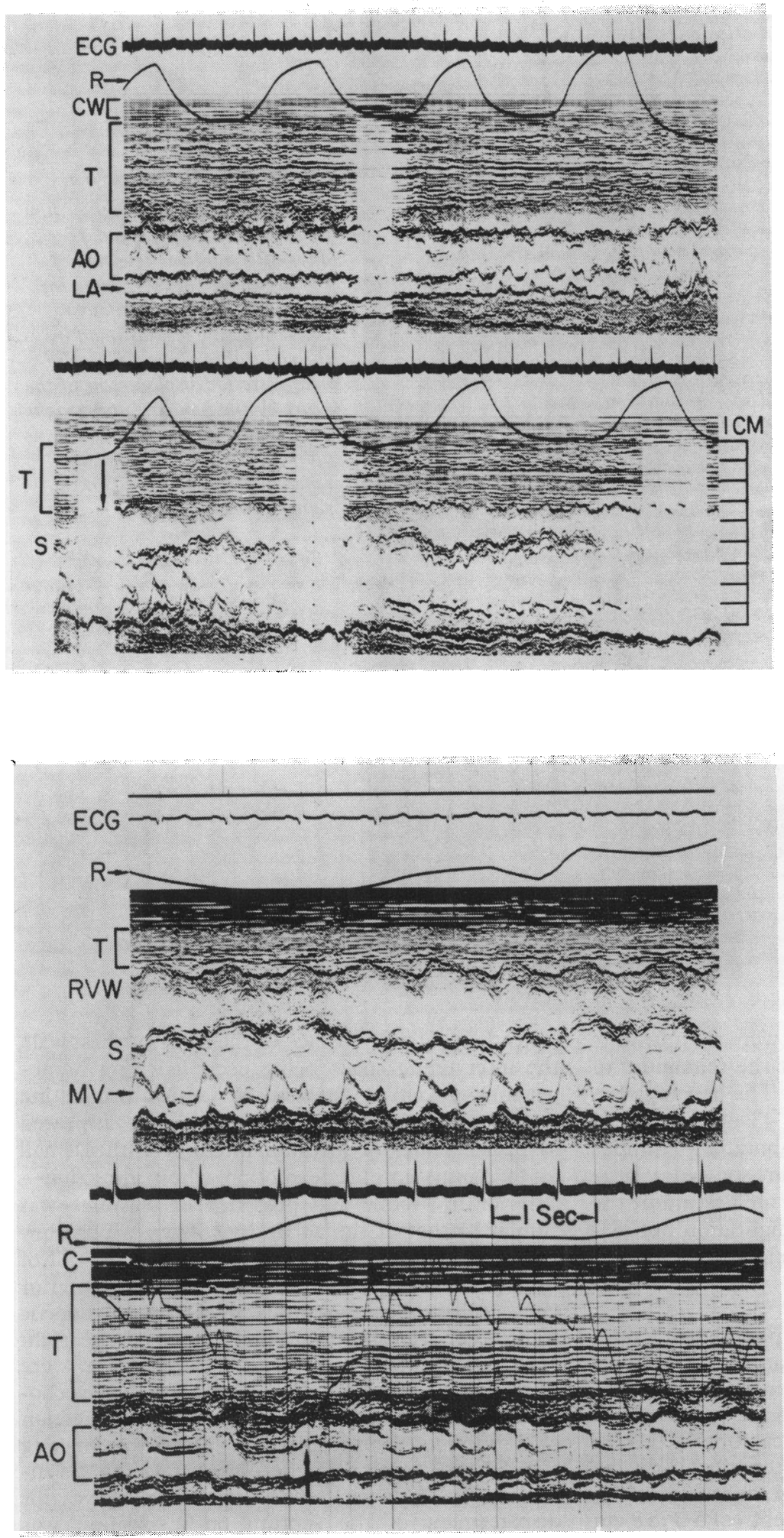

Fig. 2 Preoperative echocardiographic scan (continuous recording). The band of echoes from the tumour (T) are seen to occupy the space between the anterior wall of the right ventricle (shown by the arrow on the lower tracing) and the anterior chest wall $(C W)$. Note the progressive decrease in tumour dimension as the scan moves from the base of the heart towards the apex. See text for discussion. $E C G$, electrocardiogram, $A O$, aortic root; $L A$, left atrium; $S$, interventricular septum. The tissue depth scale is constant throughout and shown at the end of the lower tracing. In this and all subsequent tracings the respiratory trace $(R)$ moves upward on inspiration.

Fig. 3 Preoperative echocardiograms obtained with the transducer held stationary on the chest wall and pointed toward the respective valves. Upper: Note the effect of respiration $(R)$ on the position of the septum $(S)$, on the right ventricular dimension, and on the degree of 'pseudo-prolapse' of the anterior mitral valve leaflet $(M V)$. The space between the anterior wall of the right ventricle $(R V W)$ and the chest wall is occupied by the tumour $(T)$. Lower: Note the posterior displacement of the aortic root and the profound effect of respiration on the duration of ejection as measured on the aortic valve echogram. The ejection time is $\mathbf{2 4 0}$ ms on expiration, whereas on inspiration the aortic valve barely opens for $80 \mathrm{~ms}$ (black arrow). See text for discussion. $C$, external carotid pulse tracing. Other abbreviations as in Fig. 2. 
primarily responsible for an increase in right ventricular dimension at the expense of the left ventricle whose end-diastolic dimension varied from about $35 \mathrm{~mm}$ on expiration to $25 \mathrm{~mm}$ on inspiration. The mitral valve showed a prominent systolic posterior movement that became maximal on expiration (Fig. 3, upper). In the lower strip of Fig. 3, the profound effect of respiration on the volume and output of the left ventricle can be appreciated; thus, the ejection time, as measured on both the carotid pulse tracing and the aortic valve echogram (Stefadouros and Witham, 1975), varied from a maximal value of $240 \mathrm{~ms}$ on expiration (5th beat, lower strip, Fig. 3) to a minimal value of $80 \mathrm{~ms}$ on inspiration, during which the aortic valve barely opened and the carotid pulse tracing collapsed (3rd beat, lower strip, Fig. 3). Intermediate values for the ejection time were observed in other beats.

Because of increasing respiratory distress, an emergency thoractomy was carried out on the day after admission, and the greater part of the tumour was excised. It was impossible to resect it entirely because of its extension into the superior portion of the anterior mediastinum laterally and posteriorly around the great vessels, trachea, and oesophagus. The excised mass, a poorly differentiated thymocytic thymoma, was a solid tumour of $988 \mathrm{~g}$, containing a few areas of cystic degeneration. After operation the patient was initially supported by a mechanical respirator, and received a course of irradiation to the mediastinum (total dose 4800 rads), with considerable symptomatic improvement.

The postoperative chest $x$-ray film (Fig. 1, lower) showed the remaining part of the tumour in the superior mediastinum; the heart size, configuration, and position were normal. An echogram carried out on the 10th postoperative day showed the remainder of the tumour as a $1 \mathrm{~cm}$ band immediately behind the anterior wall of the chest, normal dimensions of the cardiac cavities without respiratory variation, disappearance of the mitral valve 'pseudo-prolapse' seen before operation, and a small effusion behind the posterior wall of the left atrium. The ejection time was normal and unaffected by respiration.

\section{Discussion}

The clinical syndrome of cardiac tamponade, superior vena caval obstruction, dysphagia, and respiratory distress in this patient can be completely accounted for by the mechanical compression the tumour exerted on the heart, superior vena cava, oesophagus, and trachea, respectively. Though the tumour-being obvious on the chest film-was not actually 'discovered' by $\mathrm{M}$-mode echocardiography, this technique was very useful in two respects: it demonstrated the extracardiac situation and solid nature of the tumour, and it excluded possibly coexisting cardiac lesions whose presence might have been obscured by the dramatic clinical picture produced by the tumour.

Large anterior or superior mediastinal masses are easily detectable on routine chest $x$-ray films but their differentiation from cardiomegaly or vascular tumours, and the determination of their gross anatomy usually necessitate additional radiographic, angiocardiographic, or ultrasonic studies. Ultrasound B-scan examination has been often, though not invariably (Cardello et al., 1974), successful in making the clinically important discrimination between solid and cystic mediastinal masses (Friday, 1973; Peterson et al., 1975), and similar results have been obtained with $\mathrm{A}$-mode ultrasonography (Birnholz, 1973; Goldberg, 1973). In at least two cases, $M$-mode echocardiography has been successful in defining the cystic nature of a large pericardial cyst (Friday, 1973) and a large cystic thymoma (Schloss et al., 1975) and excluding any associated heart abnormality, other than a prolapsed mitral valve found in the former case (Friday, 1973).

The echocardiographic appearance of the tumour in our patient was indicative of a solid mass. Thus, at a gain level low enough to permit visualisation of the heart cavities as echo-free spaces, discrete linear echoes of medium intensity were present at any depth within the tumour (Fig. 3). Furthermore, these echoes showed a small but distinct systolic anterior motion which was concordant and synchronous with that of the aortic root from which it was presumably transmitted. This echocardiographic pattern bears no resemblance to the echofree space observed by $M$-mode echocardiography in cases of cystic masses (Schloss et al., 1975; Peterson et al., 1975).

The echogram of the heart itself was helpful in excluding such conditions as constrictive pericarditis or pericardial effusion as a cause of the cardiac tamponade. Mechanical external compression of the heart by the tumour significantly affected the echocardiographic functional anatomy of the heart. Thus, the normal phenomenon of decreased left ventricular (and increased right ventricular) filling on inspiration was much exaggerated in this patient, resulting in a drastic inspiratory curtailment of left ventricular stroke volume and ejection time, and a prominent pulsus paradoxus. Similar, pronounced reciprocal respiratory variations in the echocardiographic dimension and hence volume of the two ventricles is a frequent finding in patients with significant pericardial effusion (unpublished observations), in whom a posterior 'pseudo-prolapse' of 
the mitral valve is also occasionally seen (Levisman and Abbasi, 1976; Vignola et al., 1976). The functional nature of this 'pseudo-prolapse' and its indirect association with the compression of the heart in these cases is established by its prompt disappearance after aspiration of the pericardial effusion (Levisman and Abbasi, 1976; Vignola et al., 1976) or, in this patient, resection of the compressing mediastinal tumour.

Our experience from this patient together with that of other investigators indicates that $M$-mode echocardiography is as useful as other noninvasive radiographic or ultrasonic techniques in the evaluation of mediastinal tumours and their mechanical effect upon the function of the heart.

\section{References}

Allee G., Logue, B., and Mansour, K. (1973). Thymic cys 1 simulating multiple cardiovascular abnormalities and presenting with pericarditis and pericardial tamponade. American fournal of Cardiology, 31, 377-380.

Birnholz, J. C. (1973). Sonic differentiation of cysts and homogeneous solid masses. Radiology, 108, 699-702.

Cardello, F. P., McQuown, D. S., and Dollinger, M. (1974). Ultrasound in diagnosis of parapericardial masses. (Letter to the Editor.) fournal of the American Medical Association, 227, 1124.

Ferrané, J., Guermonprez, J. L., Vasile, N., and Maurice, P. (1970). Silhouette cardiaque anormale et tumeurs thymiques. Fournal de Radiologie et d'Electrologie et de Médecine Nucléaire, 51, 207-216.
Friday, R. O. (1973). Paracardiac cyst: diagnosis by ultrasound and puncture. (Letter to the Editor.) Fournal of the American Medical Association, 226, 82.

Goldberg, B. B. (1973). Mediastinal ultrasonography. fournal of Clinical Ultrasound, 1, 114-119.

Kincaid, O. W., Brandenburg, R. O., and Bernatz, P. E. (1960). Experiences with angiography as a guide to mediastinal exploration. Fournal of the American Medical Association, 173, 613-624.

Levisman, J. A., and Abbasi, A. S. (1976). Abnormal motion of the mitral valve with pericardial effusion: pseudoprolapse of the mitral valve. American Heart fournal, 91, 18-20.

Peterson, D. T., Zatz, L. M., and Popp, R. L. (1975). Pericardial cyst ten years after acute pericarditis. Chest, 67, 719-721.

Schloss, M., Kronzon, I., Gelber, P. M., Reed, G. E., and Berger, A. (1975). Cystic thymoma simulating constrictive pericarditis. The role of echocardiography in the differential diagnosis. Fournal of Thoracic and Cardiovascular Surgery, 70, 143-146.

Shaver, V. C., Bailey, W. R., and Marrangoni, A. G. (1965). Acquired pulmonic stenosis due to external cardiac compression. American fournal of Cardiology, 16, 256-261.

Stefadouros, M. A., and Witham, A. C. (1975). Systolic time intervals by echocardiography. Circulation, 51, 114-117.

Vignola, P. A., Pohost, G. M., Curfman, G. D., and Myers, G. S. (1976). Correlation of echocardiographic and clinical findings in patients with pericardial effusion. American fournal of Cardiology, 37, 701-707.

Requests for reprints to Dr. Miltiadis A. Stefadouros, Section of Cardiology, Department of Medicine, Medical College of Georgia, Augusta, Georgia 30902, U.S.A.

\section{Clinical application of intra-aortic balloon pump}

A course will be held on 25-27 November 1977 at the Konover Hotel, Miami Beach, Florida. It will cover indications for use of the intra-aortic balloon pump and other circulatory assist devices for patients in cardiogenic shock.

Information can be obtained by writing to Division of Continuing Medical Education, University of Miami School of Medicine, PO Box 520875, Miami, Florida 33152, U.S.A. (Tel. A/C 305-547-6716). 